PROCEEDINGS OF THE

AMERICAN MATHEMATICAL SOCIETY

Volume 129, Number 5, Pages 1329-1334

S 0002-9939(00)05652-5

Article electronically published on October 10, 2000

\title{
ON THE INDEPENDENCE OF CORRESPONDENCES
}

\author{
XIAOAI LIN \\ (Communicated by Carl G. Jockusch, Jr.)
}

\begin{abstract}
An almost independent set-valued process on a Loeb product space is shown to be representable as the closure of a sequence of its selections which are almost independent themselves. This provides a Castaing type representation in terms of independent correspondences. Different definitions of independence for correspondences in the literature are also unified in a general setting.
\end{abstract}

\section{InTRODUCTION}

The study of set-valued analysis traces its history back to the first three decades of this century. In his 1933 book Topologie, Kuratowski gave set-valued maps their proper status. The research on the measurability of set-valued maps and their selections was initiated by Von Neumann in the 1930's. Set-valued maps, which are also known as correspondences and multifunctions, have been extensively studied in recent years, due to their rich applications in control theory, optimization, mathematical economics, stochastic analysis and so on. See Aubin-Frankowska [3], Khan-Sun [7], Matheron [10, Wagner [14] and their references.

A basic property of measurable correspondences is the so-called Castaing representation, which says that a closed valued measurable correspondence can be represented as the closure of a sequence of its selections. The first aim of this paper is to study this type of representation in the setting of independent correspondences. Note that the original version of the Castaing representation already implies that independent correspondences can be represented in terms of possibly non-independent selections. Our Theorem 1 presents an independence version of the Castaing representation that guarantees the existence of independent selections representing the correspondences.

In previous work, different definitions of independence for correspondences have been used. For example, Artstein-Hart 2 defined independent correspondences via the inverse images of closed sets in a Euclidean space $\mathbb{R}^{n}$, while Sun [13] used the inverse images of open sets in a complete separable metric space. The second aim of this paper is to unify these definitions of independent correspondences in a general context. In particular, we characterize the condition of almost independence for correspondences via inverse images of three different classes of sets in a complete

Received by the editors May 13, 1999 and, in revised form, July 8, 1999.

2000 Mathematics Subject Classification. Primary 28B20, 60 E05.

The author is grateful to an anonymous referee and Yeneng Sun for many helpful suggestions on the exposition of the paper. 
separable metric space, namely, the closed sets, the open sets and the more general Borel sets.

The plan of the paper is as follows. Section 2 contains some preliminaries, where some relevant notation and definitions are given. The results are presented and discussed in Section 3. Section 4 includes their proofs.

\section{Preliminaries}

We shall first fix some notation and definitions. A correspondence is a mapping whose values are nonempty sets. A selection of a correspondence $G$ from a probability space $(\Lambda, \Sigma, \mu)$ to a complete separable metric space $X$ is a measurable mapping $g: \Lambda \rightarrow X$ with $g(\omega) \in G(\omega)$ for almost every $\omega$ in $\Lambda$. The correspondence $G$ is said to be measurable if for any open set $O$ in $X, G^{-1}(O)$ is measurable (see [5]). Note that for a set $B$ in $X, G^{-1}(B)=\{\omega: G(\omega) \cap B \neq \emptyset\}$. The distribution of a selection $g$ of $G$ is the probability measure $\mu g^{-1}$ defined on the Borel $\sigma$-algebra $\mathcal{B}(X)$ of $X$ by $\mu g^{-1}(C)=\mu\{\omega: g(\omega) \in C\}$ for any $C \in \mathcal{B}(X)$. For easy reference, we give the definition of a Castaing representation for a closed valued correspondence (also called an almost everywhere Castaing representation). Note that a correspondence is called closed valued if it takes values in the space of closed sets in $X$.

Definition 1. A Castaing representation of a closed valued measurable correspondence $G$ from a probability space $(\Lambda, \Sigma, \mu)$ to a complete separable metric space $X$ is a sequence $\left\{g^{i}\right\}$ of selections of $G$ such that for $\mu$-almost all $\omega \in \Lambda$, the set $G(\omega)$ is the closure $\overline{\left\{g^{i}(\omega)\right\}}$ of the countable set $\left\{g^{i}(\omega): i=1,2, \ldots\right\}$.

As shown in Proposition 1.1 in [12, joint measurability and independence are not compatible with each other except for some trivial cases. One needs a suitable framework to study independence using continuum methods. For this purpose, we shall restrict our attention in this paper to correspondences on a special type of probability space, namely, Loeb spaces (see Loeb 8$]$ ). Let $(T, L(\mathcal{T}), L(\lambda))$ and $(\Omega, L(\mathcal{A}), L(P))$ be Loeb probability spaces, where $L(\mathcal{T})$ and $L(\mathcal{A})$ are $\sigma$-algebras of subsets of $T$ and $\Omega$ respectively, and $L(\lambda)$ and $L(P)$ are the corresponding countably additive probability measures on the measurable spaces $(T, L(\mathcal{T}))$ and $(\Omega, L(\mathcal{A}))$. Let $(T \times \Omega, L(\mathcal{T} \otimes \mathcal{A}), L(\lambda \otimes P))$ be a Loeb product space; it is not only an extension of the usual product measure space but also a standard probability space itself. As was first shown by Keisler, an important property of the Loeb product space is that the Fubini Theorem still holds for this special product space (see 6]). Another important property is that the incompatibility of joint measurability and independence can be settled in this larger measure-theoretic framework (see Theorem 6.2 in [1]).

For this paper, a process is a measurable function $f$ from the Loeb product space $(T \times \Omega, L(\mathcal{T} \otimes \mathcal{A}), L(\lambda \otimes P))$ to a complete separable metric space $X$. For each $t \in T$, $\omega \in \Omega, f_{t}$ and $f_{\omega}$ denote the function $f(t, \cdot)$ on $\Omega$ and $f(\cdot, \omega)$ on $T$ respectively. A set-valued process $F$ is a closed valued measurable correspondence from the Loeb product space $(T \times \Omega, L(\mathcal{T} \otimes \mathcal{A}), L(\lambda \otimes P))$ to $X$. Similarly, for each $\omega \in \Omega$ and $t \in T$, the correspondences $F_{\omega}, F_{t}$ are defined by $F_{\omega}=F(\cdot, \omega)$ and $F_{t}=F(t, \cdot)$.

Next, we give a formal definition of independent correspondences (see also [13]).

Definition 2. For a set-valued process $F$ from $(T \times \Omega, L(\mathcal{T} \otimes \mathcal{A}), L(\lambda \otimes P))$ to $X$, if for $L(\lambda \otimes \lambda)$-almost all $\left(t_{1}, t_{2}\right) \in T \times T$, the correspondences $F_{t_{1}}$ and $F_{t_{2}}$ are independent, i.e., for any open sets $O_{1}$ and $O_{2}$ in $X$, the events $F_{t_{1}}^{-1}\left(O_{1}\right)$ and 
$F_{t_{2}}^{-1}\left(O_{2}\right)$ are independent, where $F_{t_{i}}^{-1}\left(O_{i}\right)=\left\{\omega: F_{t_{i}}(\omega) \cap O_{i} \neq \emptyset\right\}$ for $i=1,2$. Then the correspondences $F_{t}$ are said to be almost surely pairwise independent.

One can also define almost mutual independence for correspondences as in [13]. However, these two almost versions of independence of correspondences are actually equivalent (see Theorem 1 in [13]). Thus, we shall refer to them simply as almost independence. Almost independence of the selections can be defined in a similar way, and the pairwise and mutual versions are equivalent as well (see Theorem 3 in [12]).

\section{THE RESUlts}

The well-known Castaing representation theorem provides a structural result for a measurable correspondence. The following theorem is an independence version of the Castaing representation, which shows the structure of an almost independent set-valued process to be rather simple, i.e., the closure of a sequence of almost independent point-valued processes. The key point in the proof is to relate a result of Artstein in [1] on a dense representation of a correspondence in terms of distributions to a result of Sun in [13] on the removal of widespread correlations through a redistribution. Once this connection is made, the proof becomes simple.

Theorem 1. Let $F$ be a set-valued process from the Loeb product space

$$
(T \times \Omega, L(\mathcal{T} \otimes \mathcal{A}), L(\lambda \otimes P))
$$

to a complete separable metric space $X$. Suppose that the correspondences $F_{t}$ are almost independent. Then, there is a sequence $\left\{f^{i}(t, \omega)\right\}$ of selections of $F$ such that for almost all $(t, \omega), F(t, \omega)=\overline{\left\{f^{i}(t, \omega)\right\}}$, and for any fixed $i$ and all $t$ in $T$, the random variables $f_{t}^{i}$ are almost independent.

In Artstein-Hart [2], correspondences are said to be independent if the events obtained from the inverse images of closed sets in a Euclidean space $\mathbb{R}^{n}$ are independent. On the other hand, the inverse images of open sets in a complete separable metric space are used in $[13$ and this paper to define independent correspondences. A natural question is whether these definitions are equivalent in terms of almost independent set-valued processes. Theorem 2 below answers this question and also provides a more general characterization in terms of Borel sets.

Theorem 2. The following versions of almost independence of correspondences $F_{t}$ are equivalent:

(i) For any Borel sets $B_{1} \subseteq X, B_{2} \subseteq X$, and $L(\lambda \otimes \lambda)$-almost all $\left(t_{1}, t_{2}\right) \in T \times T$, $F_{t_{1}}^{-1}\left(B_{1}\right)$ and $F_{t_{2}}^{-1}\left(B_{2}\right)$ are independent;

(ii) For any closed sets $C_{1} \subseteq X, C_{2} \subseteq X$, and $L(\lambda \otimes \lambda)$-almost all $\left(t_{1}, t_{2}\right) \in$ $T \times T, F_{t_{1}}^{-1}\left(C_{1}\right)$ and $F_{t_{2}}^{-1}\left(C_{2}\right)$ are independent;

(iii) For any open sets $O_{1} \subseteq X, O_{2} \subseteq X$, and $L(\lambda \otimes \lambda)$-almost all $\left(t_{1}, t_{2}\right) \in T \times T$, $F_{t_{1}}^{-1}\left(O_{1}\right)$ and $F_{t_{2}}^{-1}\left(O_{2}\right)$ are independent;

(iv) For $L(\lambda \otimes \lambda)$-almost all $\left(t_{1}, t_{2}\right) \in T \times T, F_{t_{1}}^{-1}\left(O_{1}\right)$ and $F_{t_{2}}^{-1}\left(O_{2}\right)$ are independent for any open sets $O_{1}$ and $O_{2}$ in $X$.

Note that Part (iv) above is simply Definition 2 in Section 2 and Part (ii) can also be rewritten in the same form. While it is natural to conjecture the above equivalence result, not all parts have a trivial proof. The most interesting part of the proof is to show that (iv) implies (i). One may want to use open sets to 
approximate Borel sets in $X$. However, there is no natural distribution to measure such approximations. On the other hand, we have seen above that the almost independent selections play an important role in an almost independent correspondences. The idea is to construct some special selection to relate (i) to (iv). In particular, we construct a selection $g$ of the set-valued process $F$ such that the inverse image of a Borel set under $g$ is the same as that under $F$. Then the problem on correspondences can be reduced to selections, on which many results can be applied.

\section{The PROOFS}

To prove our Theorems 1 and 2, we need Theorem 2 in 13 . For the convenience of the reader, we include that theorem as a lemma below. It says that for a given selection of an almost independent set-valued process, one can always find another selection inducing the same distribution as that of the given one and having almost independent random variables.

Lemma 1. Let $F$ be a set-valued process from the Loeb product space

$$
(T \times \Omega, L(\mathcal{T} \otimes \mathcal{A}), L(\lambda \otimes P))
$$

to a complete separable metric space $X$ such that the correspondences $F_{t}$ are almost independent. Let $\mu$ be the distribution of a selection $f$ of $F$ as a random variable on $T \times \Omega$. Then there is a selection $g$ of $F$ such that the random variables $g_{t}$ are almost independent and the distribution of $g$ viewed as a random variable on $T \times \Omega$ is $\mu$.

Proof of Theorem 1. Let $\mathcal{F}_{F}$ denote the ensemble of selections of $F$. For selections $\alpha$ and $\beta$ of $F$, let $P(\alpha, \beta)$ denote the Prohorov distance (see [4]) between distributions $L(\lambda \otimes P) \alpha^{-1}$ and $L(\lambda \otimes P) \beta^{-1}$. Since the Prohorov metric on $X$ is separable (see [4]), one can find a sequence $\left\{g^{i}\right\}_{i=1}^{\infty}$ of selections of $F$, dense in $\mathcal{F}_{F}$ with respect to $P(\cdot, \cdot)$. Such a sequence is called a $P$-dense representation of the correspondence $F$ in [1].

Since the correspondences $F_{t}$ are almost independent, Lemma 1 implies that one can find a sequence $\left\{f^{i}\right\}_{i=1}^{\infty}$ of selections of $F$ such that for each fixed $i=1,2, \cdots$, the random variables $f_{t}^{i}$ are almost independent and

$$
L(\lambda \otimes P)\left(f^{i}\right)^{-1}=L(\lambda \otimes P)\left(g^{i}\right)^{-1} .
$$

It is clear that the sequence $\left\{f^{i}\right\}_{i=1}^{\infty}$ is also a $P$-dense representation of the correspondence $F$. Proposition 2.3 in [1] says that a $P$-dense representation of $F$ is a Castaing representation of $F$. Hence $F(t, \omega)=\overline{\left\{f^{i}(t, \omega)\right\}}$ for almost all $(t, \omega)$. The theorem is thus proven.

Proof of Theorem 2. (i) $\Rightarrow$ (ii): Obvious.

(ii) $\Rightarrow$ (iii): For any open set $O_{i}, i=1,2$, there is an increasing sequence $\left\{C_{i}^{n}\right\}_{n=1}^{\infty}$ of closed sets such that $O_{i}=\bigcup_{n=1}^{\infty} C_{i}^{n}$. Then

$$
\begin{aligned}
F_{t_{i}}^{-1}\left(O_{i}\right) & =\left\{\omega: F_{t_{i}}(\omega) \cap O_{i} \neq \emptyset\right\}=\left\{\omega: F_{t_{i}}(\omega) \cap \bigcup_{n=1}^{\infty} C_{i}^{n} \neq \emptyset\right\} \\
& =\bigcup_{n=1}^{\infty}\left\{\omega: F_{t_{i}}(\omega) \cap C_{i}^{n} \neq \emptyset\right\}=\bigcup_{n=1}^{\infty} F_{t_{i}}^{-1}\left(C_{i}^{n}\right) .
\end{aligned}
$$


By (ii), there exists $A^{n} \subseteq T \times T$ such that $L(\lambda \otimes \lambda)\left(A^{n}\right)=0$, and for any $\left(t_{1}, t_{2}\right) \in T \times T-A^{n}, F_{t_{1}}^{-1}\left(C_{1}^{n}\right)$ and $F_{t_{2}}^{-1}\left(C_{2}^{n}\right)$ are independent. Let $A=\bigcup_{n=1}^{\infty} A^{n}$. Then $L(\lambda \otimes \lambda)(A)=0$. For any $\left(t_{1}, t_{2}\right) \in T \times T-A, F_{t_{1}}^{-1}\left(C_{1}^{n}\right)$ and $F_{t_{2}}^{-1}\left(C_{2}^{n}\right)$ are independent for all $n \geq 1$, and hence we have

$$
\begin{gathered}
L(P)\left(F_{t_{1}}^{-1}\left(O_{1}\right) \cap F_{t_{2}}^{-1}\left(O_{2}\right)\right)=\lim _{n \rightarrow \infty} L(P)\left(F_{t_{1}}^{-1}\left(C_{1}^{n}\right) \cap F_{t_{2}}^{-1}\left(C_{2}^{n}\right)\right) \\
=\lim _{n \rightarrow \infty} L(P)\left(F_{t_{1}}^{-1}\left(C_{1}^{n}\right)\right) \cdot L(P)\left(F_{t_{2}}^{-1}\left(C_{2}^{n}\right)\right)=L(P)\left(F_{t_{1}}^{-1}\left(O_{1}\right)\right) \cdot L(P)\left(F_{t_{2}}^{-1}\left(O_{2}\right)\right) .
\end{gathered}
$$

Thus, (ii) implies (iii).

(iii) $\Rightarrow($ iv): Since $X$ is a complete separable metric space, it has a countable base $\mathcal{B}_{0}$ which is closed under the operation of finite unions. Thus, every open set can be expressed as an increasing union of sets in $\mathcal{B}_{0}$. Using the same idea as in the proof of $(\mathrm{ii}) \Rightarrow$ (iii), we can get a subset $A$ of $T \times T$ such that $L(\lambda \otimes \lambda)(A)=0$ and for any $\left(t_{1}, t_{2}\right) \in T \times T-A, F_{t_{1}}^{-1}\left(O_{1}\right)$ and $F_{t_{2}}^{-1}\left(O_{2}\right)$ are independent for any open sets $O_{1}$ and $O_{2}$ in $X$.

We end the proof of the theorem by showing that (iv) implies (i).

(iv) $\Rightarrow(\mathbf{i})$ : For the Borel set $B_{i}$ in $X, i=1,2$, first, we shall construct a selection $g^{i}$ of $F$, such that $\left(g^{i}\right)^{-1}\left(B_{i}\right)=F^{-1}\left(B_{i}\right)$. Let $A_{i}$ denote the set $F^{-1}\left(B_{i}\right)$, i.e., $A_{i}=\left\{(t, \omega): F(t, \omega) \cap B_{i} \neq \emptyset\right\}$, and $g^{\prime}$ a selection of $F$. Let $F^{\prime}(t, \omega)$ be a correspondence defined on the space $\left(A_{i}, L(\mathcal{T} \otimes \mathcal{A}) \cap A_{i}\right), F^{\prime}(t, \omega)=F(t, \omega) \cap B_{i}$. Then the graph of $F^{\prime}$ is the intersection of the graph of $F$ with $A_{i} \times B_{i}$, which is measurable in the product $\sigma$-algebra $\left(L(\mathcal{T} \otimes \mathcal{A}) \cap A_{i}\right) \otimes \mathcal{B}\left(B_{i}\right)$. By the measurable selection theorems in [5] and [14], there exists a measurable selection $\varphi^{i}$ of $F^{\prime}$. Define a selection $g^{i}$ of $F$ as follows:

$$
g^{i}(t, \omega)= \begin{cases}\varphi^{i}(t, \omega), & (t, \omega) \in A_{i} \\ g^{\prime}(t, \omega), & (t, \omega) \in T \times \Omega-A_{i} .\end{cases}
$$

Since $g^{i}$ is a selection of $F,\left(g^{i}\right)^{-1}\left(B_{i}\right) \subseteq F^{-1}\left(B_{i}\right)$ is obvious. For any $(t, \omega) \in$ $F^{-1}\left(B_{i}\right)=A_{i}, g^{i}(t, \omega)=\varphi^{i}(t, \omega) \in F(t, \omega) \cap B_{i}$, which implies $(t, \omega) \in\left(g^{i}\right)^{-1}\left(B_{i}\right)$, so $F^{-1}\left(B_{i}\right) \subseteq\left(g^{i}\right)^{-1}\left(B_{i}\right)$. Therefore $\left(g^{i}\right)^{-1}\left(B_{i}\right)=F^{-1}\left(B_{i}\right)$.

Next, by Lemma 1 , since the correspondences $F_{t}$ are almost independent, we can find a selection $f^{i}$ of $F$, such that $L(\lambda \otimes P)\left(f^{i}\right)^{-1}=L(\lambda \otimes P)\left(g^{i}\right)^{-1}$, and the random variables $f_{t}^{i}$ are almost independent. Then

$$
L(\lambda \otimes P) F^{-1}\left(B_{i}\right)=L(\lambda \otimes P)\left(g^{i}\right)^{-1}\left(B_{i}\right)=L(\lambda \otimes P)\left(f^{i}\right)^{-1}\left(B_{i}\right)
$$

But $\left(f^{i}\right)^{-1}\left(B_{i}\right) \subseteq F^{-1}\left(B_{i}\right)$, so the two sets $\left(f^{i}\right)^{-1}\left(B_{i}\right)$ and $F^{-1}\left(B_{i}\right)$ are $L(\lambda \otimes P)$ almost equal. Because the Fubini Theorem is still valid for the Loeb product space, the events $\left(f_{t}^{i}\right)^{-1}\left(B_{i}\right)$ and $F_{t}^{-1}\left(B_{i}\right)$ are also $L(P)$-almost equal for $L(\lambda)$-almost all fixed $t \in T$.

Finally, since the processes $f^{1}$ and $f^{2}$ have almost independent random variables, Proposition 4.9 in [12] implies that for $L(\lambda \otimes \lambda)$-almost all $\left(t_{1}, t_{2}\right) \in T \times T$, the random variables $f_{t_{1}}^{1}$ and $f_{t_{2}}^{2}$ are independent. Note that the Extension Theorem (Loève 9], p. 237) implies that the independence of random variables can be equivalently defined in terms of inverse images of open sets or closed sets or Borel sets in $X$. Thus, for $L(\lambda \otimes \lambda)$-almost all $\left(t_{1}, t_{2}\right) \in T \times T$, the events $\left(f_{t_{1}}^{1}\right)^{-1}\left(B_{1}\right)$ and $\left(f_{t_{2}}^{2}\right)^{-1}\left(B_{2}\right)$ are independent, and so are the events $F_{t_{1}}^{-1}\left(B_{1}\right)$ and $F_{t_{2}}^{-1}\left(B_{2}\right)$. Thus we are done. 


\section{REFERENCES}

1. Z. Artstein, On dense univalued representations of multivalued maps, Rend. Circ. Mat. Palermo 33 (1984), 340-350. MR 87d:28011

2. Z. Artstein and S. Hart, Law of large numbers for random sets and allocation processes, Mathematics of Operations Research 6 (1981), 485-492. MR 84j:60042

3. J. P. Aubin and H. Frankowska, Set Valued Analysis, Birkhäuser, Boston, 1990. MR 91d:49001

4. P. Billingsley, Convergence of Probability Measures, Wiley, New York, 1968. MR 38:1718

5. C. Castaing and M. Valadier, Convex analysis and measurable multifuctions, Lecture Notes in Mathematics 580 (1977). MR 57:7169

6. A. E. Hurd and P. A. Loeb, An Introduction to Nonstandard Real Analysis, Academic Press, Orlando, Florida, 1985. MR 87d:03184

7. M. A. Khan and Y. N. Sun, Non-cooperative games on hyperfinite Loeb spaces, J. Math. Econ. 31 (1999), 455-492. MR 2000b:91011

8. P. A. Loeb, Conversion from nonstandard to standard measure spaces and applications in probability theory, Trans. Amer. Math. Soc. 211 (1975), 113-122. MR 52:10980

9. M. Loève, Probability Theory I, 4th edition, Springer-Verlag, New York, 1977. MR 58:3132a

10. G. Matheron, Random Sets and Integral Geometry, Wiley, London, 1975. MR 52:6828

11. Y. N. Sun, A theory of hyperfinite processes: the complete removal of individual uncertainty via exact LLN, J. Math. Econ. 29 (1998), 419-503. MR 99j:28020

12. Y. N. Sun, The almost equivalence of pairwise and mutual independence and the duality with exchangeability, Probability Theory and Related Fields 112 (1998), 425-456. CMP 99:05

13. Y. N. Sun, The complete removal of individual uncertainty: multiple optimal choices and random exchange economies, Economic Theory 14 (1999), 507-544. CMP 2000:05

14. D. H. Wagner, Survey of measurable selection theorems, SIAM J. Control and Optimization 15 (1977), 859-903. MR 58:6137]

Department of Mathematics, National University of Singapore, Singapore 119260

E-mail address: scip8206@nus.edu.sg 